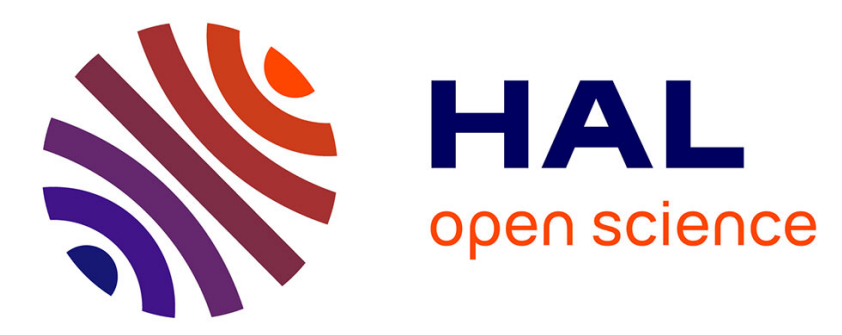

\title{
Experimental test of the crossover between the inertial and the dissipative range in a turbulent swirling flow
}

Paul Debue, Denis Kuzzay, Ewe-Wei Saw, François Daviaud, Bérengère

Dubrulle, Léonie Canet, Vincent Rossetto, Nicolás Wschebor

\section{To cite this version:}

Paul Debue, Denis Kuzzay, Ewe-Wei Saw, François Daviaud, Bérengère Dubrulle, et al.. Experimental test of the crossover between the inertial and the dissipative range in a turbulent swirling flow. Physical Review Fluids, 2018, 3 (2), pp.024602. 10.1103/PhysRevFluids.3.024602 . hal-01531233

\author{
HAL Id: hal-01531233 \\ https://hal.science/hal-01531233
}

Submitted on 26 Feb 2018

HAL is a multi-disciplinary open access archive for the deposit and dissemination of scientific research documents, whether they are published or not. The documents may come from teaching and research institutions in France or abroad, or from public or private research centers.
L'archive ouverte pluridisciplinaire HAL, est destinée au dépôt et à la diffusion de documents scientifiques de niveau recherche, publiés ou non, émanant des établissements d'enseignement et de recherche français ou étrangers, des laboratoires publics ou privés. 


\title{
Experimental test of the crossover between the inertial and the dissipative range in a turbulent swirling flow
}

\author{
Paul Debue, Denis Kuzzay, Ewe-Wei Saw, François Daviaud, and Bérengère Dubrulle \\ SPEC, CEA, CNRS, Universit Paris-Saclay, CEA Saclay, 91191 Gif-sur-Yvette, France \\ Léonie Canet and Vincent Rossetto \\ Laboratoire de Physique et Modélisation des Milieux Condensés, \\ Université Grenoble Alpes, CNRS UMR 5493, 38042 Grenoble, France \\ Nicolás Wschebor \\ Instituto de Física, Facultad de Ingeniería, Universidad de la República, J.H.y Reissig 565, 11000 Montevideo, Uruguay
}

\begin{abstract}
The kinetic energy spectrum of high-Reynolds turbulent swirling flows is experimentally studied. This spectrum, obtained from direct measurements in space, exhibits nearly two decades of Kolmogorov $k^{-5 / 3}$ decay in the inertial range of scales. Beyond this regime, in the dissipative range of scales, a crossover to a stretched exponential decay on scale $k^{2 / 3}$ is observed, in full agreement with a recent theoretical prediction based on Non-Perturbative Renormalization Group theory.
\end{abstract}

\section{INTRODUCTION}

Understanding the statistical properties of turbulence is one of the most important unsolved problems in classical physics, and is of primary interest for applications ranging from meteorology, aerodynamics, and civil engineering to astrophysics. An emblematic feature of isotropic and homogeneous turbulence in threedimensional incompressible flows is the Kolmogorov $k^{-5 / 3}$ decay of the kinetic energy spectrum [1]. This scaling law follows from Kolmogorov hypothesis of a constant energy flux in the inertial range [2, 3], that is in between (but well separated from) the energy injection (integral) scale $L$ and the dissipation (Kolmogorov) scale $\eta$, and is a very robust feature appearing in many different contexts. Conversely, theoretical predictions for Navier-Stokes (NS) turbulence, beyond Kolmogorov theory, and not on phenomenological grounds, are scarce. In particular, calculating the intermittency corrections to Kolmogorov scaling laws remains an open issue [1].

Recently, analytical predictions have been obtained for the full (space and time dependent) velocity-velocity correlation function of homogeneous and isotropic turbulence, using a field theoretic formalism and Nonperturbative Renormalisation Group (NPRG) techniques [4]. Their salient features, beside the well-known $k^{-5 / 3}$ spectrum, are i) for the two-velocity correlation function, a Gaussian dependence in the variable $k t$, where $k$ is the wave-number and $t$ the time delay in the stationary state $C(t, k) \propto \exp \left(-\alpha k^{2} t^{2}\right)$, ii) for the energy spectrum, a crossover from the Kolmogorov decay in the inertial range to a stretched exponential $E(k) \propto k^{-5 / 3} \exp \left(-\mu k^{2 / 3}\right)$ in the dissipative range. Such simple predictions can be tested both numerically and experimentally. They were indeed accurately confirmed in direct numerical simulations of NS equation in 4].

In this work, the prediction ii) is tested against experimental data, measured in von Kármán swirling flows. The data are collected over a broad range of parame- ters, including different fluids, different forcing mechanisms, and covering several decades of Reynolds number. In these experiments, the velocity measurements are directly performed in space, and not reconstructed from time measurements using Taylor's frozen turbulence hypothesis 5. They hence allow for a direct and fine determination of equal-time quantities. We show that, beyond the inertial range, the tail of the energy spectrum exhibits a clear stretched exponential behaviour on the scale $k^{2 / 3}$ in the dissipative range, providing an experimental confirmation of the theoretical prediction. The paper is organised as follows. In Sec. II, a summary of the theoretical prediction is provided. The experimental set-up is presented in Sec. III The data for the kinetic energy spectrum are analyzed in Sec. IV in the dissipative range, where the stretched exponential behaviour is observed. Further tests of universality are provided in Sec. V] and some methodological aspects are detailed in the Appendix.

\section{THEORETICAL PREDICTIONS}

Fully developed turbulence is characterized in the inertial range by the appearance of power-laws, a feature reminiscent of scale invariance, as encountered in standard critical phenomena. A turbulent state hence shares some similarities with a critical one. One of the hallmarks of critical phenomena is the emergence of fluctuations at all scales. The Renormalisation Group (RG) conceived by Wilson, is a method designed to handle such fluctuations, by performing a progressive statistical average, scale by scale, in order to compute the physical properties of the system, even at criticality [6]. In the RG method, a scale invariant (critical) system corresponds to a fixed point of RG transformations, such that the properties of the critical state can be determined from the fixed point. The RG hence appears as a natural tool to describe a turbulent state, and indeed, RG studies of turbulence 
started in the seventies [7-10] and have a long history, but they have turned out to be extremely challenging 11, 12. One of the difficulties for standard perturbative RG follows from the absence of an upper critical dimension around which the theory would be Gaussian and an usual dimension expansion could be performed.

In this context, Functional or Non-Perturbative incarnations of the RG have been proposed [13-16. Within the NPRG framework, alternative approximations are possible to avoid a dimension expansion, circumventing the difficulties of the perturbative approaches. The NPRG is based on an exact flow equation (giving the evolution with respect to the RG scale) of the effective action [17, which is the Legendre transform of the generating functional of connected correlation functions (the equivalent of Gibbs free energy in thermodynamics). This exact equation involves an infinite hierarchy of flow equations, relating the correlation functions of all orders. The standard way to exploit this equation is to devise some approximation scheme in order to truncate the hierarchy 18 20. In the context of turbulence, such schemes have been implemented for instance in [13, 14, 16], where the fixed point describing the turbulent steady state of homogeneous isotropic turbulence has been identified and studied.

Besides these studies, a major step has been achieved in [16, where it was shown that in the limit of large wave-numbers with respect to the inverse integral scale $k \gg L^{-1}$, approximations were not necessary to close the flow equations for the two-point (two-space, two-time) functions. Indeed, this closure can be achieved exactly for large wave-numbers using Ward identities, which are exact analytic relations between correlation functions deduced from the existence of symmetries. More precisely, these Ward identities are associated with extended symmetries of the NS field theory, which are time-dependent (or time-gauged) forms of global symmetries: the Galilean invariance 21, 22 and a particular shift-symmetry unveiled in 23. Such an exact closure is exceptional in the NPRG context, as well as in other approaches. The closed flow equation for the two-point correlation function of homogeneous and isotropic turbulence in the stationary state (where rotational invariance, and translational invariance in space and time on average are assumed) was established in [16, and its fixed point solution was derived in [24]. In order to write the fixed point equation, one first introduces the adimensionalized correlation function $\hat{C}$ defined by

$$
C(t, \vec{k})=\frac{\epsilon^{2 / 3}}{k^{11 / 3}} \hat{C}\left(y=\epsilon^{1 / 3} t k^{2 / 3}, x=(k / \kappa)^{2 / 3}\right),
$$

in units where $\nu=\rho=1$. In this expression, $\epsilon$ is the mean energy injection rate per unit mass, and $\kappa$ is the inverse of the RG scale. The fixed point equation for $\hat{C}$ takes a very simple form in the limit of large wavenumbers $k \gg \kappa$ and small time differences $t \ll \kappa^{-2 / 3}$, which is

$$
\partial_{x} \hat{C}(y, x)=-\alpha y^{2} \hat{C}(y, x)
$$

In this equation, $\alpha$ is a non-universal constant, whose expression can be found in 4. It cannot be determined from the fixed point equation (2) alone, but requires to solve the full flow equation (including the small wavenumber sector). The general solution of Eq. (2) provides the full space-time dependent correlation function. It reads for the original function $C(t, \vec{k})$ 24]

$$
C(t, \vec{k})=c \frac{\epsilon^{2 / 3}}{k^{11 / 3}} \exp \left[-\tilde{\alpha} t^{2} k^{2}\right]
$$

where $c$ is a non-universal constant and $\tilde{\alpha}$ is proportional to $\alpha$ with dimensional constants absorbed. The leading term in wave-number, i.e. the exponent in the exponential, is calculated exactly, the sub-leading term, i.e. the power-law, is not. The solution (3) predicts a Gaussian dependence in the variable $k t$, which indicates a violation of standard scale invariance, pertaining to intermittency. Indeed, the dynamical exponent for NS in $d=3$ is $z=2 / 3$, such that standard scaling theory would yield a $t k^{z}$ dependence of the scaling function. This result, amounting to an effective dynamical exponent $z=1$, is physically related to the sweeping effect [24].

The kinetic energy spectrum can be deduced as the "naive" equal-time $t \rightarrow 0$ limit of (3) which yields

$$
E(k)=4 \pi k^{2} C(t=0, k) \propto \epsilon^{2 / 3} k^{-5 / 3} .
$$

One hence recovers the Kolmogorov result. As mentioned above, the power-law is not exact in this calculation. Indeed, it was shown in 24 that intermittency corrections are suppressed for equal-time quantities, for which they are sub-leading in wave-numbers. They are hence not included in the equation (2), which is obtained in the large wave-number limit. On the other hand, even at equal times, this equation contains an interesting prediction beyond Kolmogorov theory, since it allows to describe the dissipative range of scales. It was shown in 24 that when the Kolmogorov scale (equivalently the Kolmogorov time) is finite, the limit $t \rightarrow 0$ must be taken with care in Eq. (2), and the appropriate limit yields the following result for the spectrum

$$
E(k)=4 \pi c \frac{\epsilon^{2 / 3}}{k^{5 / 3}} \exp \left[-\mu k^{2 / 3}\right],
$$

where $\mu$ is a non-universal constant given by

$$
\mu=\alpha \frac{\eta^{4 / 3}}{L^{2 / 3}} \sim \eta^{2 / 3} R e^{-1 / 2},
$$

where $\eta$ is the Kolmogorov scale and Re the Reynolds number. The expression (5) for the energy spectrum hence predicts a crossover from Kolmogorov $k^{-5 / 3}$ decay in the inertial range to a stretched exponential decay in the dissipative range, on scale $k^{2 / 3}$. As already emphasized, $\mu$ cannot be determined from the solution of the fixed point equation (2) alone. However, it can be estimated from experimental or numerical data. In particular, a comparison was achieved with the outcome 
of direct numerical simulations of isotropic and homogeneous turbulence based on a pseudo-spectral integration code of NS equation 24]. These simulations confirmed the stretched exponential decay in the dissipative range with a coefficient $\mu$ of order $\mu \eta^{-2 / 3} \sim 4$ to 5 for different Reynolds number $R e$. The aim of this paper is now to test this prediction in experiments.

\section{EXPERIMENTS}

The theoretical predictions have been compared with experimental results obtained in the von Kármán experimental setup in Saclay. It is made of a cylindrical tank of height $H=20 \mathrm{~cm}$ and radius $R=10 \mathrm{~cm}$ filled with water or a water-glycerol mixture, which is stirred by two counter-rotating impellers driven by two independent motors. These impellers are actually disks with eight 2-cm-high curved blades. The experimental data used in this article were obtained with these impellers counter-rotating in the direction indicated on figure 1 . conventionally called negative direction (Section III and IV), and in the opposite direction, called positive (Section V). When rotating in the negative direction, the stirring is stronger and the energy dissipation was measured to be higher than for the positive direction. The average flow generated is made of two counter-rotating toroidal cells in which the liquid is both rotating in the azimuthal direction (blue motion on figure 1) and being pumped in meridian planes (red motion). These two cells are separated by a mixing layer, about equidistant from both impellers. The von Kármán flow is characterized by a Reynolds number $R e=\frac{2 \pi R^{2} F}{\nu}$ where $F$ is the rotation frequency of the impellers and $\nu$ the kinematic viscosity of the liquid. Transition to turbulence happens around $R e=1000$, the flow is fully turbulent beyond $R e=3500$ and values as high as $R e=3.10^{5}$ can be reached in our setup [25, 26]. The setup is thermalized (at $20^{\circ} \mathrm{C}$ ) to keep the density $\rho$ and viscosity $\nu$ constant.

The torques $C_{1}$ and $C_{2}$ applied to the flow by the impellers and their frequency $F$ can be measured with SCAIME torque meters. After removing the contribution of mechanical frictions they can be used to compute the energy input rate $P$ per unit mass in the flow as :

$$
P=\frac{2 \pi F\left(C_{1}+C_{2}\right)}{\rho \pi H R^{2}}=\epsilon .
$$

This measurement also provides the global energy dissipation rate $\epsilon$, since in stationary state the injected and dissipated powers are equal. The dissipation scale can then be deduced : $\eta=\left(\nu^{3} / \epsilon\right)^{1 / 4}$. By modifying the frequency $F$ and the viscosity $\nu$ (which depends on the glycerol fraction of the mixture), it is possible to vary the dissipation scale. Performing different runs with different mixtures, we are thus able to observe different parts of the inertial range and even the beginning of the dissipation range, in a single setup and with a limited camera resolution 28. In this article, we thus consider

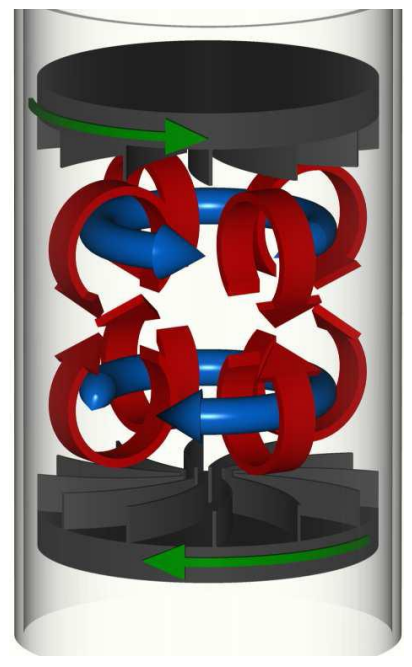

FIG. 1. Representation of the experimental setup and of the mean flow : in blue, the azimuthal motion and in red, the pumping motion

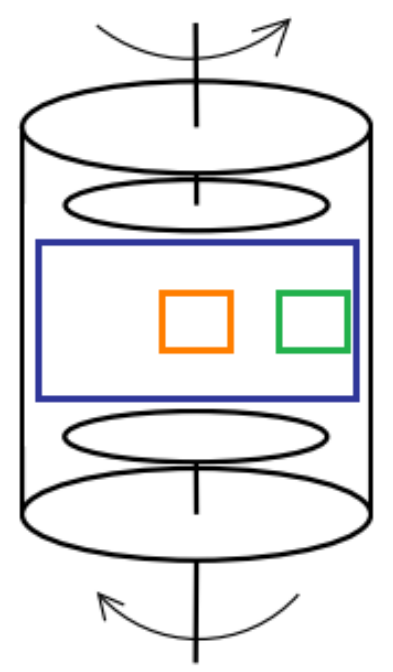

FIG. 2. Velocity measurement areas for case A (blue) and cases B, C, D and E (orange). The green area corresponds to location were turbulence is less isotropic, and is used to test universality.

in the negative direction, five configurations of the von Kármán flow, obtained with three different frequencies, two different viscosities and three different resolutions. The parameters of these five cases are gathered in Table II. In Section V] we also consider other measurements with glycerol $59 \%$ mixture, in the positive direction, to achieve lower values of $\epsilon$ at Reynolds number $R e \sim 10^{3}$ to $10^{4}$, in order to explore universality properties of the dissipative range. 


\begin{tabular}{||c||c|c|c|c|c|c|c|c|c|c||}
\hline \hline Case & $\mathrm{F}(\mathrm{Hz})$ & Glycerol volume fraction & $R e$ & $\epsilon$ & $\eta(\mathrm{mm})$ & $\lambda_{\nu}(\mathrm{mm})$ & $\lambda_{L T}(\mathrm{~mm})$ & $\Delta x(\mathrm{~mm})$ & Samples & Symbol \\
\hline \hline $\mathrm{A}$ & 5 & $0 \%$ & $3 \times 10^{5}$ & 0.046 & 0.016 & 0.39 & 0.91 & 2.4 & 29999 & $\circ$ \\
$\mathrm{B}$ & 5 & $0 \%$ & $3 \times 10^{5}$ & 0.046 & 0.016 & 0.39 & 0.91 & 0.48 & 29228 & $\square$ \\
$\mathrm{C}$ & 5 & $0 \%$ & $3 \times 10^{5}$ & 0.046 & 0.016 & 0.39 & 0.91 & 0.24 & 28000 & $\diamond$ \\
$\mathrm{D}$ & 1 & $0 \%$ & $6 \times 10^{4}$ & 0.046 & 0.074 & 1.3 & 2.0 & 0.48 & 9999 & $\Delta$ \\
$\mathrm{E}$ & 1.2 & $59 \%$ & $6 \times 10^{3}$ & 0.050 & 0.32 & 7.3 & 6.5 & 0.24 & 30188 & $\star$ \\
\hline \hline
\end{tabular}

TABLE I. Parameter space describing the 5 cases considered in counter-rotation in the negative sense. Re is the Reynolds number, $\epsilon$ the dimensionless global energy dissipation (in units of $R^{2}(2 \pi F)^{3}$ ) measured using torque-meters, $\Delta x$ is the resolution of our measurements, and $\eta$ is the Kolmogorov scale. $\lambda_{\nu}(\mathrm{mm})$ and $\lambda_{L T}$ are respectively the inner viscous and Liepmann-Taylor length scales, defined in [27. The last two columns indicate the number of velocity field samples on which the quantities were averaged and the symbol representing data from the corresponding case in all the figures of this paper.

The velocity measurements were achieved with a Stereoscopic Particle Image Velocimetry (SPIV) system which allows to get the three components of the velocity on a meridian plane of the tank. The measurement areas are shown on figure 2 : for case $\mathrm{A}$, the velocity is measured on a whole meridian plane, of width $200 \mathrm{~mm}$ and height $140 \mathrm{~mm}$ (blue area on figure 2); for cases B to E, it is measured on a limited area located at the center of this meridian plane, of width $40 \mathrm{~mm}$ and height $30 \mathrm{~mm}$ (orange area on the figure). This area is in the shear layer, it is far from the boundaries, and the mean velocity in it is small (less than 50\% the rms velocity). Such an area is also invariant by two symmetries of the set-up, i.e. the rotations of $\pi$ radians around the vertical and horizontal axes of the measurement plane intersecting in the center of the cylinder. Thus, we expect that it is the area where the turbulence is the most homogeneous and isotropic, although not rigorously (see e.g. [29]). Due to the presence of the large scale shearing motions, there are nevertheless two effects that may presumably distort the spectra, with respect to the ideal case [30]: sweeping motions, representing the advection of the small scales by the large scales, and straining motions, representing the distortion of vortices due to local shear. The first effect does not change the form of the energy spectrum [12, 30]. The second effect was discussed in depth in 31 in the case of the von Karman geometry and was shown to lead to a steepening of the slope towards -2 at scales of the order of 10 to $1 \mathrm{~cm}$. In the present paper, we consider much smaller scales, and show that the predictions of the NPRG are valid at those scales, suggesting that neither sweeping nor straining influence the spectra at small scales. In Section V, we explore further the universality of the NPRG prediction, by considering measurements over a window of width $40 \mathrm{~mm}$ and height $30 \mathrm{~mm}$ taken in the mid plane near the cylinder boundary (green area on figure 2). This corresponds to a place where the turbulence is less isotropic, as it is influenced by the boundary.

Both SPIV cameras, of resolution 1,600x1,200 pixels, were equipped with zoom lenses to focus on these areas. The reconstruction is done using peak correlation performed over $50 \%$ overlapping windows of size $16 \times 16$ pixels, so that we get a velocity field on a grid of step varying from one case to another from about $0.25 \mathrm{~mm}$ to $2.5 \mathrm{~mm}$. For each case, 10,000 or 30,000 velocity fields were acquired at frequency $15 \mathrm{~Hz}$. Thus, consecutive frames are uncorrelated and the fastest scales are statistically sampled.

For each velocity field of each case, we computed the spatial Fourier spectrum, defined as:

$$
E(k)=\int \widehat{\mathbf{u}}^{2} k^{2} d \Omega,
$$

where $\widehat{\mathbf{u}}$ is the $3 \mathrm{D}$ Fourier transform of the velocity $\mathbf{u}$. We also compute the spatial wavelet spectrum, defined as:

$$
\tilde{E}(\mathbf{x}, \ell)=\frac{\tilde{u}(\mathbf{x}, \ell)^{2}+\tilde{v}(\mathbf{x}, \ell)^{2}+\tilde{w}(\mathbf{x}, \ell)^{2}}{2},
$$

where $u, v$ and $w$ are the components of the velocity and where ${ }^{\sim}$ stands for the wavelet transform, defined as

$$
\tilde{u}(\mathbf{x}, \ell)=\int \phi\left(\frac{\mathbf{x}-\mathbf{y}}{\ell}\right) u(\mathbf{y}) d^{2} \mathbf{y} .
$$

Here, $\phi$ is the wavelet function. We used the mexican hat wavelet : $\phi(\mathbf{x}) \propto\left(1-\mathbf{x}^{2}\right) e^{-\mathbf{x}^{2} / 2}$.

As mentioned previously, the von Kármán flow is not rigorously isotropic and homogeneous, even at the center of the cylinder, as assumed in section II to derive the spectrum formula. However, we found that the slopes of the three separated components spectra are the same for a given case, only the prefactor varies, as can be seen in the appendix or on figure 5 for case E. Therefore, we believe that it makes sense to test the NPRG theory on our data, and in the sequel, we only plotted the total spectra. 
The wavelet spectra are then averaged over space (over $\mathbf{x})$. Both wavelet and Fourier spectra are further averaged over the 10,000 or 30,000 frames to get the spectra plotted on figure 3 and 4 . On these figures, we plotted, in logarithmic coordinates, the spectra normalized by $\epsilon^{2 / 3} \cdot \eta^{-5 / 3}$ as a function of $\eta / \ell$ or $\eta k$. Indeed, according to Kolmogorov theory, these quantities are linked by a universal function at least in the inertial range [1]. For comparison, we also plotted in Figure 3 the Fourier energy spectrum of the JHU database [32, 33, corresponding to a direct numerical simulation of isotropic turbulence with (dimensionless) parameters: $R e \approx 10^{5}$, $\epsilon=0.103$ and $\eta=0.00280$. We see that it agrees well with our experimental data points.

The wavelet and Fourier spectra are very close to each other, at least in the inertial range where they both behave as a power law of exponent $-5 / 3$. It can indeed be shown that the wavelet spectrum at $\ell$ is an average of the Fourier spectrum over several wavenumbers with major contribution, for our wavelet, at wavenumber $k \approx 1.6 / \ell$ (see the Appendix). This explains why the wavelet spectra show less variations and thus allow a better identification of power law behaviors.

Computing spectra from PIV data allows us to get spatial spectra without Taylor frozen turbulence hypothesis. However, the noise is stronger than with hot wire anemometry and PIV comes along with a low-pass filtering effect. We took care of considering only the wave vectors ranges in which the spectra are not affected by these effects, as shown in the Appendix.

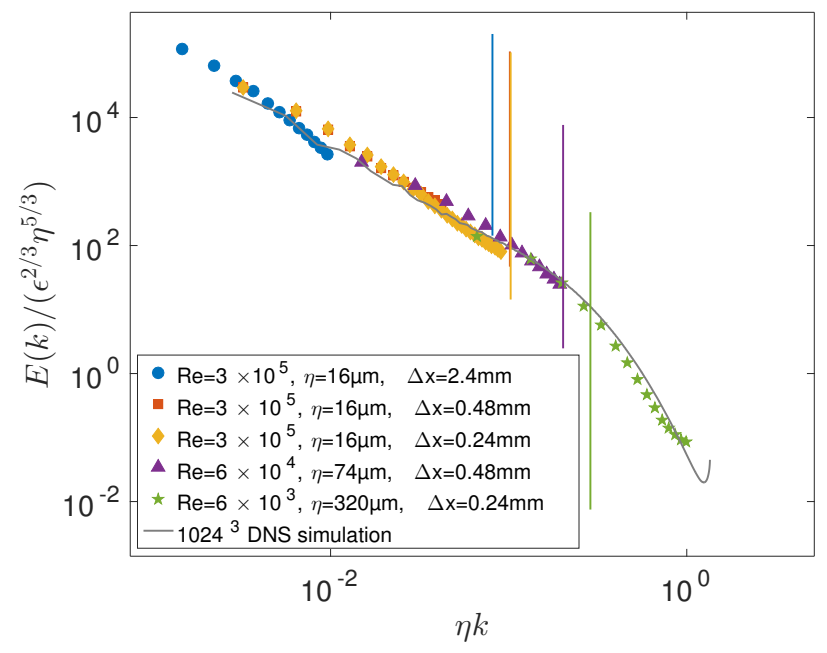

FIG. 3. Fourier spectrum in universal representation, after adimensionalization by $\epsilon$, the global energy dissipation, and $\eta$, the Kolmogorov scale. The symbols are the experimental points, with vertical lines marking out the Taylor scale $\lambda$. The continuous greyline is a numerical spectrum, obtained from a direct numerical simulation of Navier-Stokes equations in a fully periodic box of size $1024^{3}$. Data obtained from the JHTDB at http://turbulence.pha.jhu.edu.

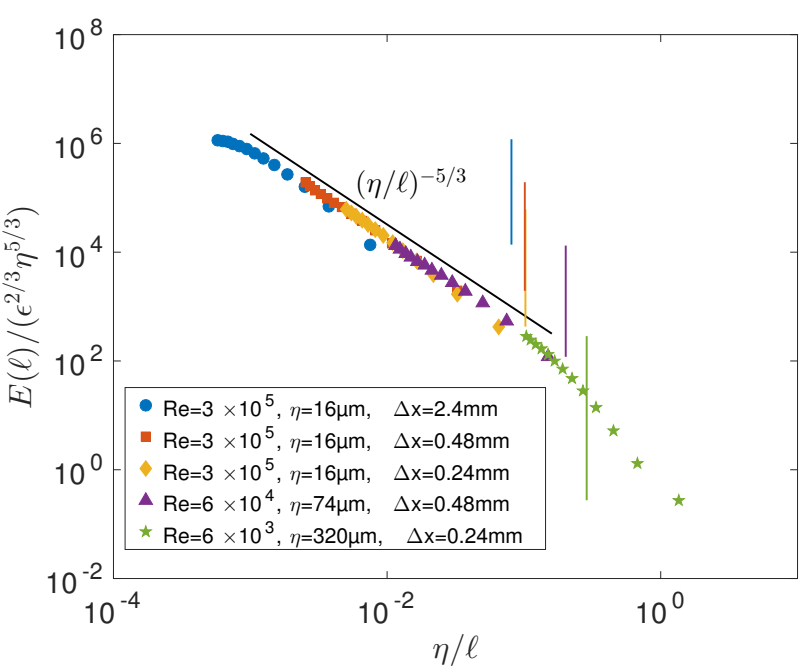

FIG. 4. Wavelet spectrum in universal representation, after adimensionalization by $\epsilon$, the global energy dissipation, and $\eta$, the Kolmogorov scale.

\section{ANALYSIS OF THE ENERGY SPECTRUM AT CROSS-OVER SCALES}

In this section and the next one, we analyze the spectrum obtained experimentally, focusing on the crossover from the inertial to the dissipative range. This crossover begins around the Taylor scale $\lambda$ defined by

$$
\lambda=\sqrt{\frac{15 \nu \overline{u^{\prime 2}}}{\epsilon}}
$$

above which viscous effects have an important effect on the vortices dynamics. The only case providing resolved scales smaller than this scale is case E, corresponding to $R e=6.10^{3}$ (green color on the plots).

Such a Reynolds number is quite low, and does not ensure separation of the energy-containing scales and the dissipation scales (see [27). Indeed, as can be seen in table I the Liepmann-Taylor length scale is smaller than the inner viscous one for case $\mathrm{E}$. However, the derivation of the spectrum expression with the NPRG in section II only assumes $k \gg L^{-1}$. For case $\mathrm{E}, k$ is always greater than $20 L^{-1}$ so we can still use this case to test the NPRG result.

The cross-over range is also the range where the socalled bottleneck effect can possibly be observed. However, our data are not relevant to describe such effect as none of the cases covers the whole bottleneck zone. This would require PIV data covering over 2 decades for a single case, which represents an experimental challenge (see Appendix). This effect is not present in the NPRG calculation either since it is a sub-leading correction in wave-number.

In order to test the existence of the stretched exponential described by Eq. (5), the energy spectrum is multiplied by $k^{5 / 3}$ and plotted in semi-logarithmic coordinates, 
the wave-numbers $k$ being represented in a $k^{2 / 3}$ scale. Fig. 6 is thus obtained. The Taylor microscales are materialized by vertical straightlines. In this representation, the inertial range data appear as short plateaus above Taylor microscale. These plateaus are more visible in log-log coordinate, see Fig. 7. Beyond these plateaus, a decade of a stretched exponential behavior on scale $k^{2 / 3}$ is visible in Fig. 6 manifest as a straight line. The estimated slope of this line, which is the coefficient in the exponential, is $\mu \simeq 6$. It is the same for the separate spectra of each velocity component, as shown in Fig. 5 . confirming the fact that anisotropy only concerns the prefactor of the spectra. This $\mu$ coefficient turns out to be comparable to previous estimates from numerical simulations [4. Indeed, the numerical Fourier spectrum, reported on Fig. 6] is close to our experimental measurements.

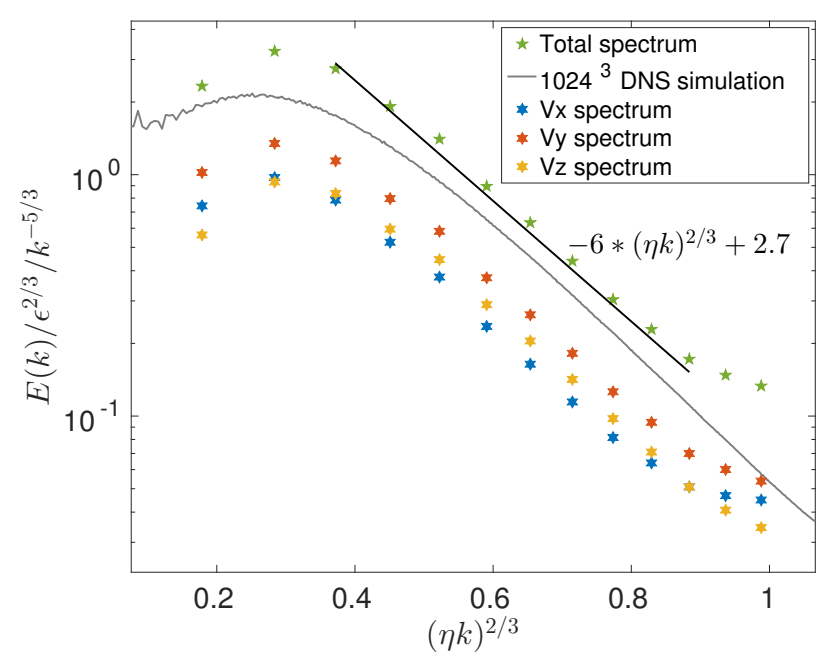

FIG. 5. Energy spectrum for each velocity component in case E, with vertical axis multiplied by $k^{5 / 3}$, and horizontal axis rescaled to $k^{2 / 3}$, plotted in log-lin scale. A stretched exponential is apparent in the dissipative range. The grey line is the spectrum of the JHU database. The black line is a fit of the spectrum according to Eq. (5)

To test the robustness of this result, we also attempted a two-parameter fit, looking for the best exponent $\alpha$ such that $\log \left(E(k) / \epsilon^{2 / 3} / k^{-5 / 3}\right)$ is an affine function of $(\eta k)^{\alpha}$. We found that $\alpha$ was in the range [0.4:0.7], becoming lower when fitting only on the higher wavenumber points. It must be stressed that the fit was done only on 7 points, making it therefore difficult to achieve a good accuracy as $\alpha$ was very sensitive to the number of points used.

For the wavelet spectra, we observe the same plateaus in log-log coordinate, Fig. 9. They are better defined than in the Fourier case. Testing the stretched exponential behavior, we also observe it in Figure 8, with $\mu=4.4$.

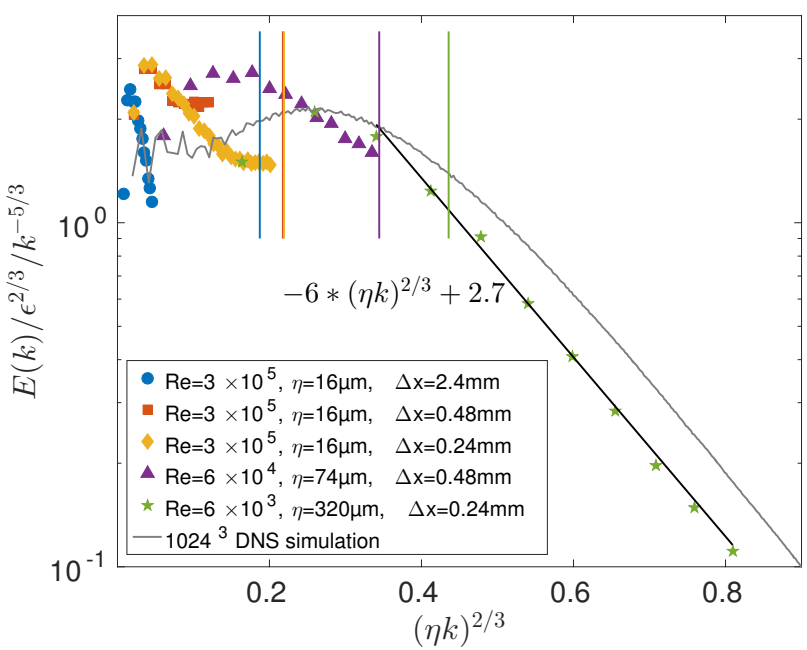

FIG. 6. Same energy spectrum as in Fig. 3, with vertical axis multiplied by $k^{5 / 3}$, and horizontal axis rescaled to $k^{2 / 3}$, plotted in log-lin scale. A stretched exponential is apparent in the dissipative range. The grey line is the spectrum of the JHU database. The black line is a fit of the spectrum according to Eq. [5].

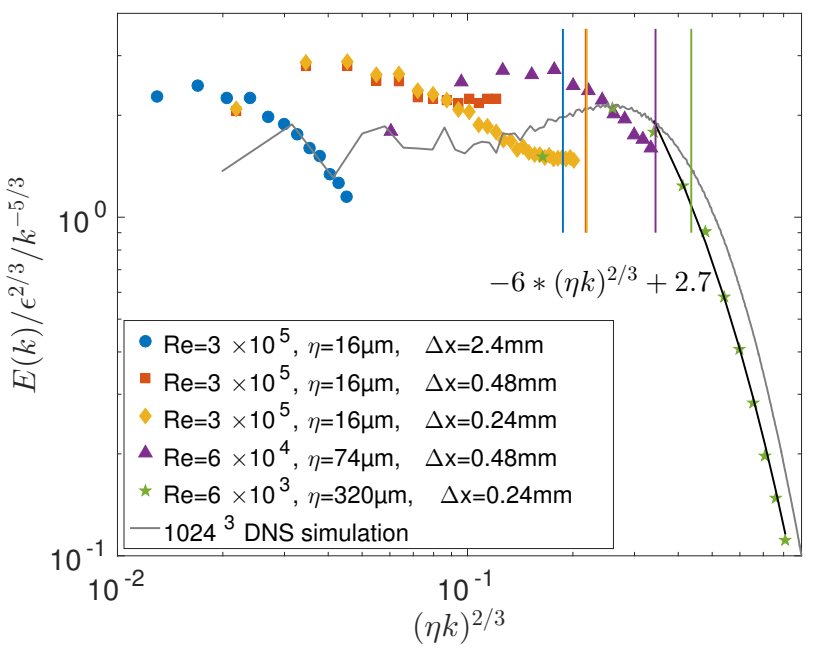

FIG. 7. Same energy spectrum as in Fig. 3, with vertical axis multiplied by $k^{5 / 3}$, and horizontal axis rescaled to $k^{2 / 3}$, plotted in log-log scale. A stretched exponential is apparent in the dissipative range. The grey line is the spectrum of the JHU database. The black line is a fit of the spectrum according to Eq. (5).

\section{UNIVERSALITY OF DISSIPATIVE RANGE}

To test the universality of the dissipative range, we have further analyzed additional experimental data, at Reynolds number between 6000 and $2.5 \times 10^{4}$, with different forcing conditions (negative and positive, and at different location (center versus near cylinder boundary), 


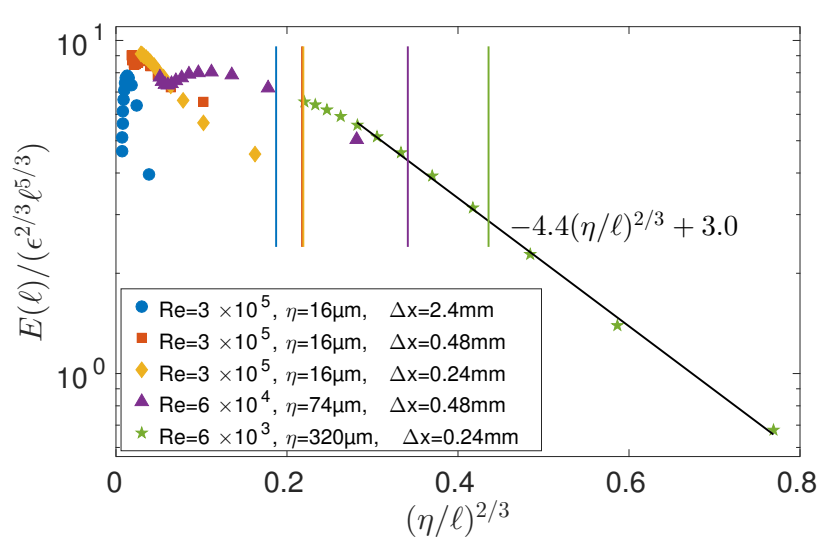

FIG. 8. Same energy spectrum as in Fig. 4, with vertical axis multiplied by $k^{5 / 3}$, and horizontal axis rescaled to $k^{2 / 3}$, plotted in log-lin scale. A stretched exponential is apparent in the dissipative range. The black line is a fit of the spectrum according to Eq. 5.

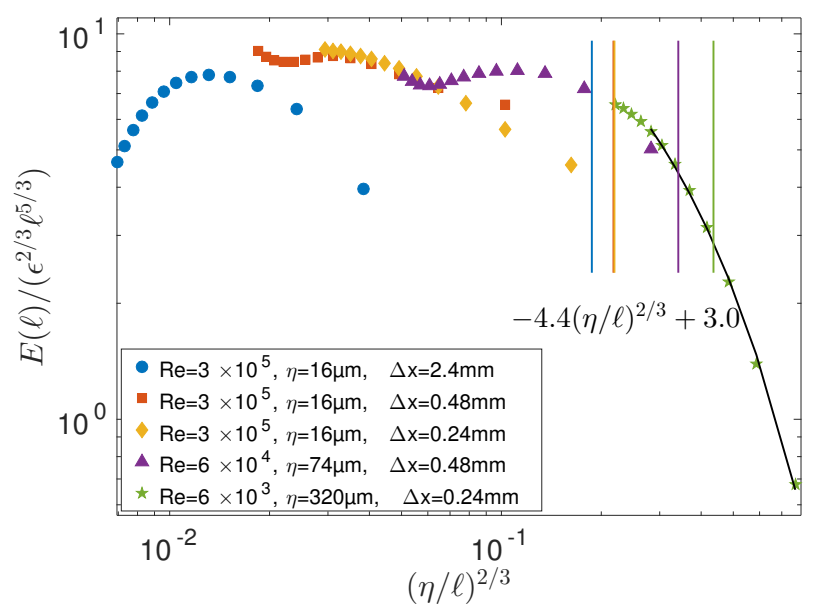

FIG. 9. Same energy spectrum as in Fig. 4, with vertical axis multiplied by $k^{5 / 3}$, and horizontal axis rescaled to $k^{2 / 3}$, plotted in log-log scale. A stretched exponential is apparent in the dissipative range. The black line is a fit of the spectrum according to Eq. (5).

keeping the same resolution. This allows to vary independently $\epsilon, \eta$ and the isotropy of the turbulence. The resulting spectra are computed, and all correspond to the dissipative range. They are gathered on Fig. 10, using the same representation as in Fig. 6. The data appear to collapse on two separate curves, that are merely shifted by a factor 2 , with respect to each other. This shift may be understood by noting that the von Kármán flow is not homogeneous, and that the energy dissipation at the center and near the cylinder boundary are not equal to each other 34. Therefore, the global energy estimate using torque measurements (which we used to collapse the data) does not necessary reproduce the local energy dissipation, that may be measured e.g. using scaling of structure functions 35. The two curves are nevertheless parallel to each other, and follow the same slope as the numerical data of JHU, with $\mu \approx 5.75$.

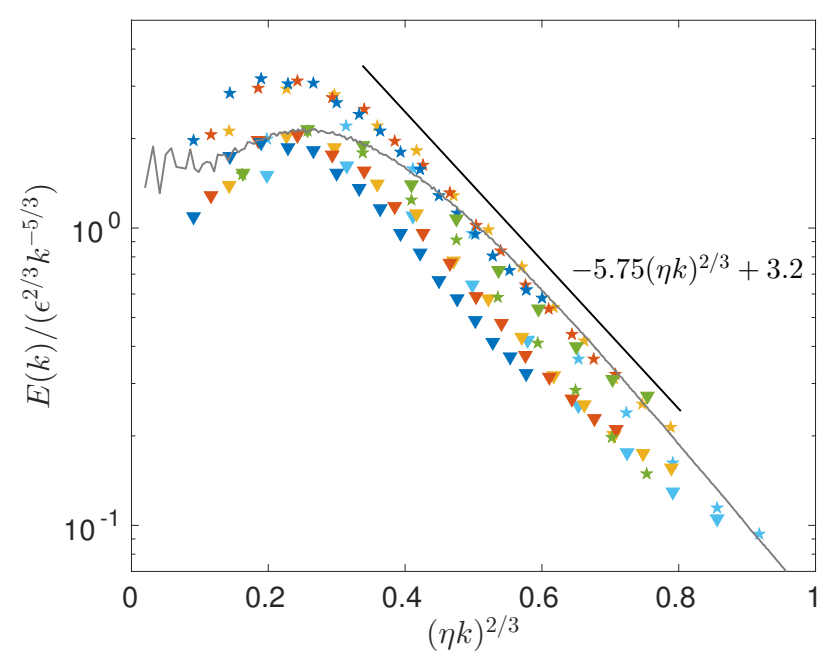

\footnotetext{
* $\mathrm{Re}=5 \times 10^{3}, \eta=0.43 \mathrm{~mm}, \quad \Delta \mathrm{x}=0.24 \mathrm{~mm}$, weaker forcing

$\star \mathrm{Re}=1 \times 10^{4}, \eta=0.26 \mathrm{~mm}, \Delta \mathrm{x}=0.24 \mathrm{~mm}$, weaker forcing

$\star \mathrm{Re}=1,5 \times 10^{4}, \eta=0.19 \mathrm{~mm}, \Delta \mathrm{x}=0.24 \mathrm{~mm}$, weaker forcing

* $\mathrm{Re}=2,5 \times 10^{4}, \eta=0.13 \mathrm{~mm}, \quad \Delta \mathrm{x}=0.24 \mathrm{~mm}$, weaker forcing

$\nabla \mathrm{Re}=5 \times 10^{3}, \eta=0.43 \mathrm{~mm}, \quad \Delta \mathrm{x}=0.24 \mathrm{~mm}$, weaker forcing, less isotropic

$\nabla \mathrm{Re}=1 \times 10^{4}, \eta=0.26 \mathrm{~mm}, \quad \Delta \mathrm{x}=0.24 \mathrm{~mm}$, weaker forcing, less isotropic

$\nabla \mathrm{Re}=1,5 \times 10^{4}, \eta=0.19 \mathrm{~mm}, \quad \Delta \mathrm{x}=0.24 \mathrm{~mm}$, weaker forcing, less isotropic

$\nabla \mathrm{Re}=2,5 \times 10^{4}, \eta=0.13 \mathrm{~mm}, \Delta \mathrm{x}=0.24 \mathrm{~mm}$, weaker forcing, less isotropic

* $\mathrm{Re}=6 \times 10^{3}, \eta=0.32 \mathrm{~mm}, \quad \Delta \mathrm{x}=0.24 \mathrm{~mm}$

$\nabla \mathrm{Re}=6 \times 10^{3}, \eta=0.32 \mathrm{~mm}, \quad \Delta \mathrm{x}=0.24 \mathrm{~mm}$, less isotropic

$-1024^{3}$ DNS simulation of John Hopkins University
}

FIG. 10. Test of universality. The energy spectrum corresponding to different forcings, different Reynolds numbers and different isotropy conditions are plotted on the same representation as in Fig 6. with vertical axis multiplied by $k^{5 / 3}$, and horizontal axis rescaled to $k^{2 / 3}$, plotted in log-lin scale. A stretched exponential is apparent in the dissipative range. The grey line is the spectrum of the JHU database. The black line is a fit of the spectrum according to Eq. (5).

Regarding the large variation of different situations, it then appear than the law predicted by the nonperturbative renormalization group is quite universal. A last check of this can be made by fitting a stretched exponential on the data for $\eta k \in[0.19 ; 0.65]$ with a two parameter fit providing the exponent of the stretched exponential $\alpha$ and the prefactor $\mu$. The best exponents are defined as those for which the sum of the residual norms was the smallest.

We thus found that the best exponent are in the range 
$[0.6,0.8]$. For example, for $\alpha \approx 0.75$ and $\mu \approx 5.5$, the average Pearson coefficient was $r=0.998563$. For $\alpha=2 / 3$ (the prediction of the NPRG formalism) and $\mu=$ 5.75 , the average Pearson coefficient is then $r=0.998507$ and the average residual norm is only $2 \%$ higher.

\section{CONCLUSION}

In this work, we analyzed extended experimental data of von Kármán swirling turbulent flows in order to test a theoretical prediction. These data were collected from a large amount of measurements, corresponding to different conditions (nature of the liquid, forcing, boundary conditions) and spanning a wide range of Reynolds numbers and anisotropy conditions. The velocities are acquired directly in space, providing an accurate determination of equal-time quantities, such as the kinetic energy spectrum. Even though our measurements were collected in conditions were the isotropy and homogeneity conditions were only approximately or not at all satisfied, the agreement of the behavior of the experimental energy spectrum with the theoretical prediction is remarkable. It exhibits a crossover from the Kolmogorov power law $k^{5 / 3}$ to a stretched exponential decay on the scale $k^{2 / 3}$, confirming that the predictions 4] based on NPRG formalism, is more universal and robust that maybe previously thought.

This opens promising perspectives, both from an experimental and a theoretical point of views. An interesting experimental development would be to resolve both the space and time dependence of the velocity-velocity correlation function. This would allow to test the functional form of the spatiotemporal correlation function predicted in [4]. On the theoretical side, a similar NPRG analysis could be applied to study two-dimensional turbulence. Another important issue to be addressed is the determination of intermittency exponents using the same formalism.

\section{ACKNOWLEDGMENTS}

This work has been supported by EuHIT, a project funded by the European Community Framework Programme 7, grant agreement no. 312778, by Labex PALM (ANR-10-LABX-0039-PALM) and by ANR EXPLOIT (ANR-16-CE06-0006-01). NW also acknowledge support from the grant no. 412 of the CSIC (UdelaR) commision.

\section{APPENDIX: METHODS}

\section{Computation of Fourier spectra}

We computed the Fourier spectra with a matlab toolobox called pivmat, developed by Frédéric Moisy and available at http://www.fast.u-psud.fr/pivmat/. To get proper spectra from PIV velocity fields, we also had to remove the higher wave vector ranges.

Indeed, a velocity field measured by PIV is close to the real velocity field averaged on square windows (of side $0.25 \mathrm{~mm}$ to $2.5 \mathrm{~mm}$ in our case), that is a smoothed velocity field. PIV has thus a cut-off effect on the spectrum. Also, PIV comes along with a noise level, which is higher than hot wire anemometry and can reach the energy of the smallest structures. These two effects counterbalance each other to some extent but one must be aware of them to distinguish the part of a spectrum which is correct from the one which is heavily affected by them and must be ignored. According to 36, the $3 \mathrm{~dB}$ cut-off

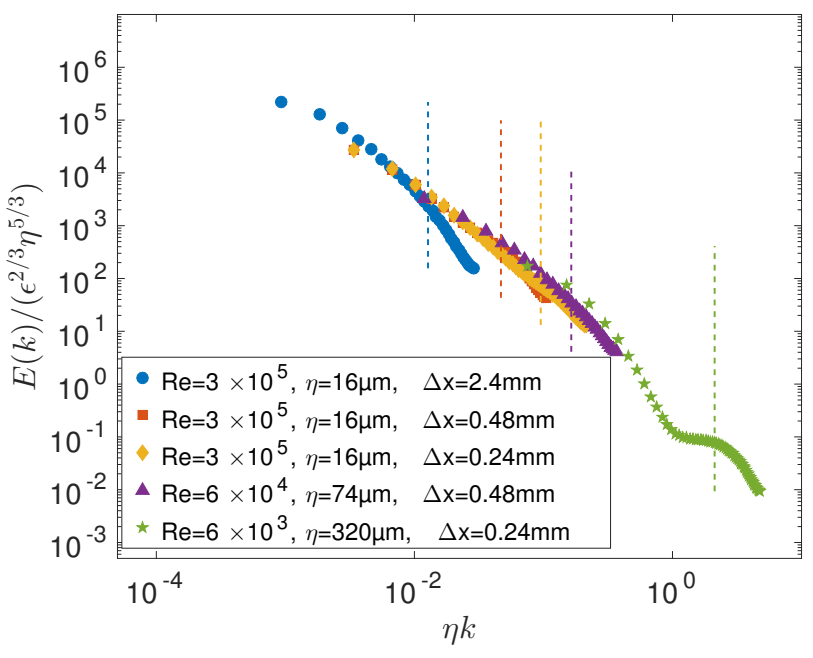

FIG. 11. Fourier spectrum in standard loglog representation with spurious parts. The vertical dashed lines refer to the cutoff wavenumber due to the PIV method. For the spectrum at $R e=6.10^{3}$, we can see that the saturation due to PIV noise happens for $k<k_{\max }$.

wavenumber due to the PIV reconstruction technique is given by $k_{\max }=2.8 / d x$ where $d x$ is the side of the interrogation windows. Note that according to Shannon's criterion, the maximum wavenumber for which the spectrum can be measured is $\pi / d x$ : the low-pass filtering effect does not reduce too much the minimum resolved scale. Figure 11 shows the whole Fourier spectra and the corresponding $k_{\max }$, represented by vertical lines. We can see the low-pass filtering effect on the spectra, and the parts which have been removed to get figure 3 . We

also see the effect of noise, which tends to counterbalance the cut-off. In the first four cases, the influence of noise is only felt for wavenumbers greater than $k_{\max }$. However, in the last case $\left(\operatorname{Re}=6.10^{3}\right)$, the spectrum reaches the noise level below $k_{\max }$. Therefore, we have cut this spectrum below $k_{\max }$, at $\eta \cdot k=1$ to get the spectrum of figure 3 . 


\section{Quantification of anisotropy of the spectra}

If the von Kármán flow at the center of the cylinder were exactly homogeneous and isotropic, the spectra of the different velocity components would collapse on a single curve. As can be seen in figures 12 to 15 and 5 this is not the case : at the largest scale (case A), the three spectra differ markedly, while at smaller scales (case B to E) only the spectra in the $x$ and $z$ component collapse, while the spectrum of the $y$ component is larger. However, in case B to E, the slopes corresponding to different components are all equal for a given case, so that they only differ by the prefactor (corresponding to different $r m s$ values). Therefore, we only showed the total spectra in the figures of this paper.

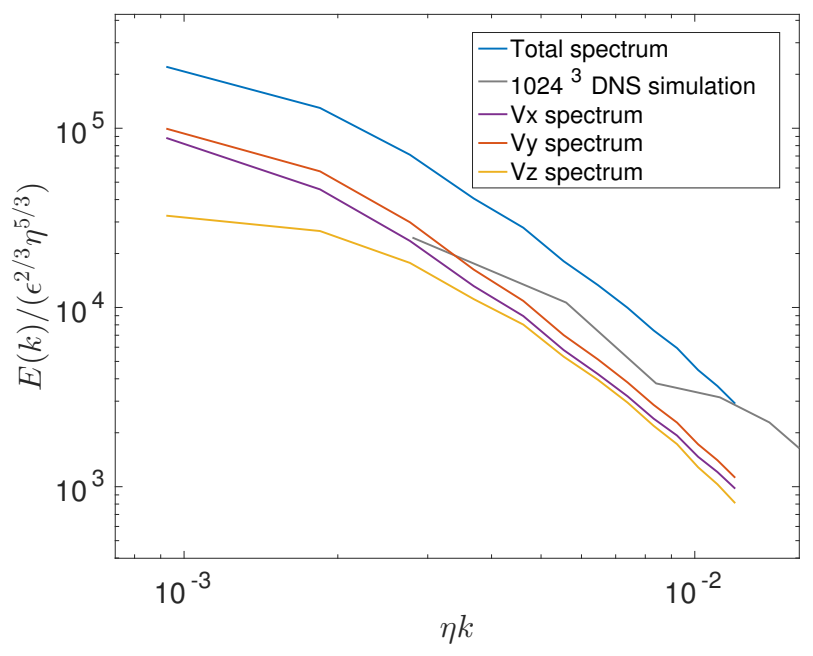

FIG. 12. Total Fourier spectrum and Fourier spectra by components for case A.

\section{Link between Fourier and wavelet spectrum}

We chose to plot wavelet spectra along with Fourier spectra because they are smoother and show more clearly the power laws. Here, we detail the link between Fourier and wavelet spectra. A wavelet spectrum is a local spectrum, computed for each $\mathbf{x}$. It allows to detect space variations of the fluctuations. To get a global spectrum, we average it over $\mathbf{x}$. It can be shown that the wavelet global spectrum is an average of the Fourier spectrum over k [37] :

$$
\tilde{E}(\ell) \propto \int E(k)|\widehat{\phi}(\ell k)|^{2} d k
$$

( $\widehat{\phi}$ is the Fourier transform of the wavelet $\phi$ defined in section III Thus, the wavelet spectrum is smoother than the Fourier spectrum. In this formula, the most important contribution of the sum is obtained for $k \cdot \ell=k_{\phi}$

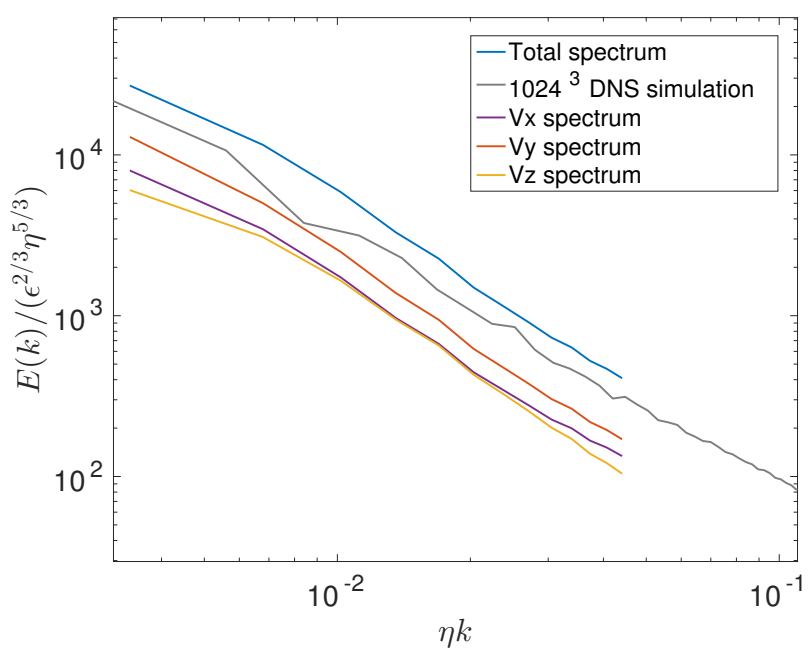

FIG. 13. Total Fourier spectrum and Fourier spectra by components for case B.

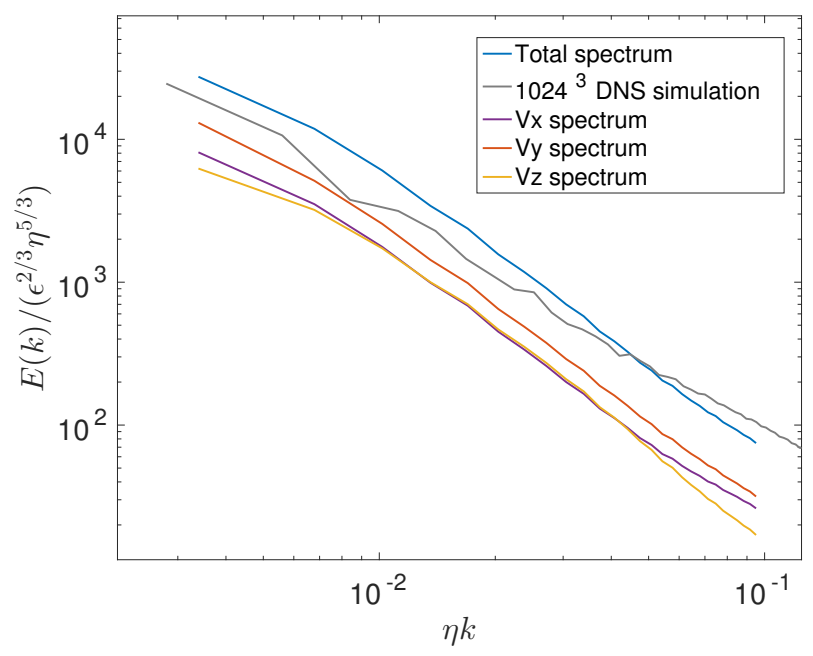

FIG. 14. Total Fourier spectrum and Fourier spectra by components for case C.

( $k_{\phi}$ is dimensionless) such that $\left|\widehat{\phi}\left(k_{\phi}\right)\right|=\max _{k^{\prime}}\left(\left|\widehat{\phi}\left(k^{\prime}\right)\right|\right)$, so that we can say that the wavelet spectrum at $\ell$ corresponds to the Fourier spectrum at $k=k_{\phi} / \ell$. For our wavelet, $k_{\phi} \approx 1.6$. In this paper, we computed the wavelet spectra at scales $n \cdot d x$ with $n=1$ to 13 . Thus, the smallest scale is still greater than the PIV cut-off scale and the scale at which the noise level becomes too important. 


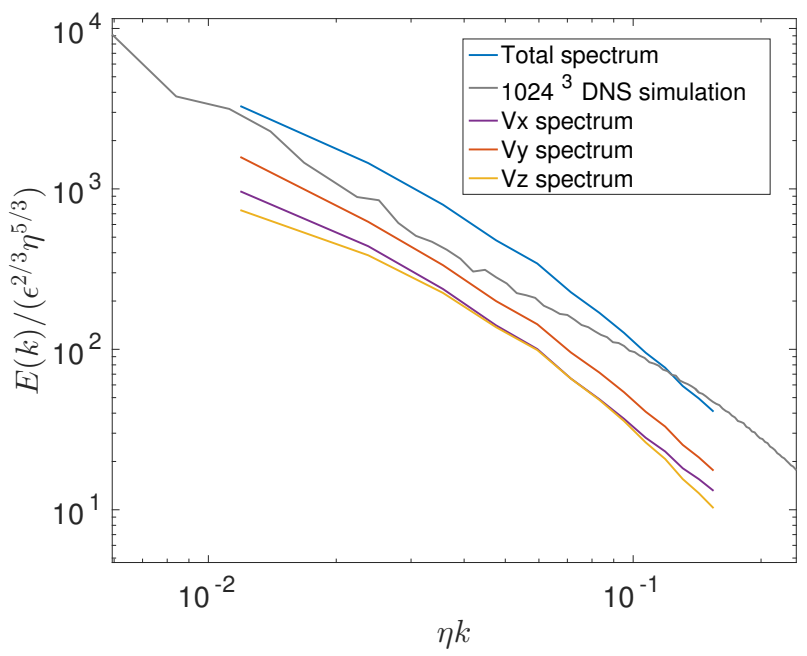

FIG. 15. Total Fourier spectrum and Fourier spectra by components for case D.

[1] U. Frisch, Turbulence: the legacy of A. N. Kolmogorov (Cambridge University Press, Cambridge, 1995).

[2] A. N. Kolmogorov, "The local structure of turbulence in incompressible viscous fluid for very large Reynolds number," Dokl. Akad. Nauk SSSR 30, 299 (1941).

[3] A. N. Kolmogorov, "The local structure of turbulence in incompressible viscous fluid for very large reynolds numbers," Proceedings of the Royal Society of London A: Mathematical, Physical and Engineering Sciences 434, 9-13 (1991)

[4] Léonie Canet, Vincent Rossetto, Nicolás Wschebor, and Guillaume Balarac, "Spatiotemporal velocity-velocity correlation function in fully developed turbulence," arXiv:1607.03098 (2016).

[5] G. I. Taylor, "The spectrum of turbulence," Proc. Roy. Soc. Lond. A , 476-490 (1938).

[6] K. G. Wilson and J. Kogut, "The Renormalization Group and the $\epsilon$-expansion," Phys. Rep. C 12, 75 (1974).

[7] Dieter Forster, David R. Nelson, and Michael J. Stephen, "Long-time tails and the large-eddy behavior of a randomly stirred fluid," Phys. Rev. Lett. 36, 867-870 (1976).

[8] Dieter Forster, David R. Nelson, and Michael J. Stephen, "Large-distance and long-time properties of a randomly stirred fluid," Phys. Rev. A 16, 732-749 (1977).

[9] C. DeDominicis and P. C. Martin, "Energy spectra of certain randomly-stirred fluids," Phys. Rev. A 19, 419$422(1979)$

[10] J. D. Fournier and U. Frisch, "Remarks on the renormalization group in statistical fluid dynamics," Phys. Rev. A 28, 1000-1002 (1983)

[11] L. Ts. Adzhemyan, N. V. Antonov, and A. N. Vasil'ev, The Field Theoretic Renormalization Group in Fully Developed Turbulence (Gordon and Breach, London, 1999).
[12] Ye Zhou, "Renormalization Group theory for fluid and plasma turbulence," Phys. Rep. 488, 1 - 49 (2010).

[13] P. Tomassini, "An exact Renormalization Group analysis of 3D well developed turbulence," Phys. Lett. B 411, 117 (1997).

[14] Carlos Mejía-Monasterio and Paolo MuratoreGinanneschi, "Nonperturbative Renormalization Group study of the stochastic Navier-Stokes equation," Phys. Rev. E 86, 016315 (2012).

[15] Andrei A Fedorenko, Pierre Le Doussal, and Kay Jrg Wiese, "Functional Renormalization Group approach to decaying turbulence," J. Stat. Mech.: Theor. and Exp. 2013, P04014 (2013).

[16] Léonie Canet, Bertrand Delamotte, and Nicolás Wschebor, "Fully developed isotropic turbulence: Nonperturbative renormalization group formalism and fixed-point solution," Phys. Rev. E 93, 063101 (2016)

[17] Christof Wetterich, "Exact evolution equation for the effective potential," Phys. Lett. B 301, 90 - 94 (1993)

[18] Jurgen Berges, Nikolaos Tetradis, and Christof Wetterich, "Non-perturbative Renormalization flow in quantum field theory and statistical physics," Phys. Rep. 363, 223 - 386 (2002), arXiv:hep-ph/0005122

[19] B. Delamotte, An introduction to the Non-Perturbative Renormalization Group in Renormalization Group and Effective Field Theory Approaches to Many-Body Systems, edited by J. Polonyi and A. Schwenk, Lecture Notes in Physics (Springer, Berlin, 2012).

[20] Peter Kopietz, Lorenz Bartosch, and Florian Schütz, Introduction to the Functional Renormalization Group, Lecture Notes in Physics (Springer, Berlin, 2010).

[21] N. V. Antonov, S. V. Borisenok, and V. I. Girina, "Renormalisation Group approach in the theory of fully developed turbulence. Composite operators of canonical 
dimension 8," Theor. Math. Phys. 106, 75 (1996).

[22] A. Berera and D. Hochberg, "Gauge symmetry and Slavnov-Taylor identities for randomly stirred fluids," Phys. Rev. Lett. 99, 254501 (2007).

[23] Léonie Canet, Bertrand Delamotte, and Nicolás Wschebor, "Fully developed isotropic turbulence: Symmetries and exact identities," Phys. Rev. E 91, 053004 (2015).

[24] Léonie Canet, Vincent Rossetto, Nicolás Wschebor, and Guillaume Balarac, "Spatiotemporal velocity-velocity correlation function in fully developed turbulence," Phys. Rev. E 95, 023107 (2017)

[25] F Ravelet, A Chiffaudel, and F Daviaud, "Supercritical transition to turbulence in an inertially-driven von karman closed flow," Journal of Fluid Mechanics 601, 339-364 (2008)

[26] B Saint-Michel, B Dubrulle, L Mari, F Ravelet, and F Daviaud, "Evidence for forcing-dependent steady states in a turbulent swirling flow," Phys. Rev. Lett. 111 (2013).

[27] Y Zhou, "Unification and extension of the similarity scaling criteria and mixing transition for studying astrophysics using high energy density laboratory experiments or numerical simulations," Physics Plasmas 14, 082701 (2007).

[28] E W Saw, D Kuzzay, D Faranda, A Guittonneau, F Daviaud, C Wiertel-Gasquet, V Padilla, and B Dubrulle, "Experimental characterization of extreme events of inertial dissipation in a turbulent swirling flow," Nature Communications 7 (2016)

[29] N Ouellette, $\mathrm{H} \mathrm{Xu}, \mathrm{M}$ Bourgoin, and E Bodenschatz, "Small-scale anisotropy in lagrangian turbulence," New
Journal Of Physics 8 (2006).

[30] Y Zhou, W H Matthaeus, and P Dmitruk, "Magnetohydrodynamic turbulence and time scales in astrophysical and space plasmas," Rev. Mod. Phys. 76, 1015 (2007).

[31] E Herbert, F Daviaud, B Dubrulle, S Nazarenko, and A Naso, "A dual non-Kolmogorov cascades in a von Karman flow," EPL 100 (2012).

[32] Y. Li, E. Perlman, M. Wan, Y. Yang, R. Burns, C. Meneveau, S. Chen, A. Szalay, and G. Eyink, "A public turbulence database cluster and applications to study lagrangian evolution of velocity increments in turbulence," Journal of Turbulence 9 (2008).

[33] E. Perlman, R. Burns, Y. Li, and C. Meneveau, "Data exploration of turbulence simulations using a database cluster," Supercomputing SC07, ACM, IEEE (2007).

[34] D Kuzzay, D Faranda, and B Dubrulle, "Global vs local energy dissipation: The energy cycle of the von karman flow," Phys. Fluids 27 (2015)

[35] E W Saw, P Debue, D Kuzzay, F Daviaud, and B Dubrulle, "On the universality of anomalous scaling exponents of structure functions in turbulent flows," J. Fluid Mechanics submitted (2017).

[36] J M Foucaut, J Carlier, and M Stanislas, "Piv optimization for the study of turbulent flow using spectral analysis," Measurement Science and Technology 15, 1046 (2004).

[37] M Farge and K Schneider, "Wavelets : application to turbulence," Encyclopedia of Mathematical Physics , 408 (2006). 